\title{
Ordering infinite utility streams comes at the cost of a non-Ramsey set
}

\author{
Luc Lauwers
}

\author{
Center for Economic Studies, K.U.Leuven \\ Naamsestraat 69, B-3000 Leuven, BELGIUM \\ Luc.Lauwers@econ.kuleuven.be \\ Phone: 016.32.68.09. Fax: 016.32.67.96 *
}

February 5, 2009

\begin{abstract}
The existence of a Paretian and finitely anonymous ordering in the set of infinite utility streams implies the existence of a non-Ramsey set (a nonconstructive object whose existence requires the axiom of choice). Therefore, each Paretian and finitely anonymous quasi-ordering either is incomplete or does not have an explicit description. Hence, the possibility results of Svensson (1980) and of Bossert, Sprumont, and Suzumura (2006) do require the axiom of choice.
\end{abstract}

JEL classification: D60; D70; D90

Keywords: Intergenerational justice; Pareto; Multi-period social choice; Axiom of choice; Constructivism.

\section{Introduction}

With respect to the social objective in his infinite horizon growth model, Ramsey (1928a, p543) argues

*I am deeply indebted to Marc Fleurbaey who guided me into this problem already in 1996 . I thank Marc for the many discussions. I also thank Norbert Brunner, Geert Dhaene, Johan Quaegebeur, Frans Spinnewyn, Peter Vallentyne, and Luc Van Liedekerke for helpful conversations. This paper was presented in the workshop "Intergenerational equity in climate negotiations, overlapping generations and social welfare" (CORE, April 2006) and at "The eighth international meeting of the society for social choice and welfare" (Istanbul, July 2006). This text presents research results of the Belgian Programme of Interuniversity Poles of Attraction initiated under the Science Policy Programmes of the Prime Minister's Office, Belgium. The scientific responsibility is assumed by its authors. 
... that we do not discount later enjoyments in comparison with earlier ones, a practice which is ethically indefensible and arises merely from the weakness of the imagination ...

This basic principle of treating generations equally was already defended by Sidgwick (1907) and is formally expressed by the anonymity condition. In the literature on repeated games, the anonymity principle captures the idea of patience or of no discounting (e.g. Rubinstein (1979) and Fudenberg and Tirole (1991, Chapter 5). A second basic principle, known as the Pareto principle, attaches positive value to the well-being of generations.

Both principles - anonymity and Pareto - are widely accepted (see Basu and Mitra, 2007; and Bossert, Sprumont, and Suzumura, 2006). The question whether or not an ordering $^{1}$ can respect the two principles (and how such an ordering looks like) has received a lot of attention. Diamond (1965) established the celebrated result that finite anonymity, ${ }^{2}$ Pareto, and continuity are incompatible when ordering infinite utility streams. Basu and Mitra (2003) show that Diamond's impossibility result persists when continuity is dropped and representability is imposed: a Paretian social welfare function does not satisfy anonymity and is necessarily inequitable. Since representability excludes (Rawlsian) lexicographic relations, one is invited to check the robustness of Diamond's result when both continuity and representability are dropped. Here, Svensson (1980) proves the possibility result that there exists a Paretian and finitely anonymous ordering. Recent work in this strand includes Bossert, Sprumont, and Suzumura (2006), which extends Svensson result: there exist finitely anonymous and Paretian orderings that satisfy a suitable version of the Pigou-Dalton principle (or Hammond's equity principle). These possibility results, however, rely on nonconstructive methods (the Axiom of Choice; Szpilrajn, 1930) and therefore lose a lot of attractiveness. ${ }^{3}$ In Hermann Weyl's words: a nonconstructive existence proof informs the world that a treasure exists without disclosing its location.

Given these tracks - incompatibilities versus possibilities through the Axiom of Choice -Fleurbaey and Michel (2003, p794) conjecture that

there is no explicit (that is, avoiding the axiom of choice or similar contrivances) description of an ordering which satisfies weak Pareto and indifference to finite permutations.

The question, thus, is: Is it possible to construct an ordering in the set of infinite utility streams that satisfies finite anonymity and Pareto?

This note confirms (a discrete version of) the Fleurbaey-Michel-conjecture. The main result (Section 4, Theorem) can be phrased as follows:

\footnotetext{
${ }^{1}$ A quasi-ordering is a transitive and reflexive binary relation. An ordering is a complete quasi-ordering.

${ }^{2}$ Finite anonymity restricts the application of the standard anonymity requirement to situations where utility streams differ in at most a finite number of components. In a setting involving infinite utility streams, finite anonymity is considered a weak intergenerational equity principle (Fleurbaey and Michel, 2003; Basu and Mitra, 2007).

${ }^{3}$ In case the existence of an object requires the Axiom of Choice, then we say that the object "is nonconstructive" or "does not have an explicit description".
} 
Theorem. A finitely anonymous and Paretian quasi-ordering in the set of infinite utility streams made up of zeros and ones either is incomplete, or is a nonconstructive object (and hence has no explicit description).

In the spirit of Weyl: the world is informed about a treasure (a finitely anonymous and Paretian ordering) and about the fact that - with certainty - no one will ever disclose its location.

In order to obtain this result, I consider the restricted domain $\{0,1\}^{\mathbb{N}_{0}}$ of zero-one-utility streams and I show that the existence of a finitely anonymous and Paretian ${ }^{4}$ ordering in this domain entails the existence of a non-Ramsey set. From Mathias (1977) we know that a non-Ramsey set (Ramsey, 1928b) is a nonconstructive object. The result extends to the standard domain $[0,1]^{\mathbb{N}_{0}}$ studied by Diamond, Svensson and Basu and Mitra. Indeed, the restriction of a finitely anonymous and Paretian ordering in $[0,1]^{\mathbb{N}_{0}}$ to the set $\{0,1\}^{\mathbb{N}_{0}}$ is a finitely anonymous and Paretian ordering in the restricted domain $\{0,1\}^{\mathbb{N}_{0}}$. In other words, the existence of a finitely anonymous and Paretian ordering in $[0,1]^{\mathbb{N}_{0}}$ entails the existence of a finitely anonymous and Paretian ordering in $\{0,1\}^{\mathbb{N}_{0}}$.

This result is in line with those obtained by Zame (2007). Zame works with the standard domain $[0,1]^{\mathbb{N}_{0}}$, observes that finite anonymity boils down to the exchangeability of certain random variables, applies the Hewitt-Savage 0-1 law, and shows that the existence of a finitely anonymous and Paretian ordering entails the existence of a nonmeasurable set which also is a nonconstructive object (Mathias, 1977).

The next section collects preliminaries. Section 3 shows how to use ultrafilters on the set of positive integers in order to obtain finitely anonymous and Paretian orderings. Section 4 recalls the definition of a Ramsey set and proves the Theorem. The end of this section positions our result against those obtained by Zame (2007). The different results are compared on the basis of the domain $\left([0,1]^{\mathbb{N}_{0}}\right.$ versus $\left.\{0,1\}^{N}\right)$ and the particular version of the Pareto axiom. In the domain $[0,1]^{\mathbb{N}_{0}}$ our result is slightly weaker than the one obtained by Zame. In the restricted domain $\{0,1\}^{\mathbb{N}_{0}}$ of zero-one-utility streams our result is slightly stronger than the one obtained by Zame.

\section{Preliminaries}

We introduce basic definitions (e.g. Bossert et al, 2006; Basu and Mitra, 2007) and we recall the Axiom of Choice.

\subsection{Basic definitions}

Let $\mathbb{N}_{0}=\{1,2,3, \ldots\}$ denote the set of positive integers and $\mathbb{R}$ the set of real numbers. Let $Y \subseteq \mathbb{R}$ be the set of all possible utility levels. We assume that $Y$ has at least two

\footnotetext{
${ }^{4}$ The Pareto axiom in the theorem is labeled "intermediate Pareto" and assumes sensitivity in each infinite set of coordinates. Intermediate Pareto is stronger than weak Pareto and weaker than strong Pareto (see Section 2).
} 
distinct elements, say, 0 and 1 . The set $X=Y^{\mathbb{N}_{0}}$ collects all possible utility streams and is called the domain. An infinite utility stream $x$ is a vector in $X$. Each $x$ in $X$ can be viewed as a map from $\mathbb{N}_{0}$ to $Y$, associating with each $n$ in $\mathbb{N}_{0}$ the element $x_{n}$ in $Y$. Each utility stream $x$ in $\{0,1\}^{\mathbb{N}_{0}}$ is identified with the subset $\left\{n \mid x_{n}=1\right\}$ of $\mathbb{N}_{0}$. Let $\mathcal{S}$ collect all subsets of $\mathbb{N}_{0}$. Due to the identification of subsets of $\mathbb{N}_{0}$ with their indicator functions, we abuse language and say that $\mathcal{S}$ is a subset of $X$. Vector inequalities are denoted $\leq,<$, and $\ll$. Set inclusions are denoted $\subseteq$ and $\subset$.

A quasi-ordering is a reflexive and transitive binary relation and an ordering is a complete quasi-ordering. The symmetric and the asymmetric component of the quasi-ordering $\precsim$ are denoted by $\sim$ and $\prec$. The relation $\precsim_{1}$ is a subrelation to the relation $\precsim_{2}$ if for each $x$ and $y$ in $X$ we have $(i) x \precsim_{1} y$ implies $x \precsim_{2} y$ and $(i i) x \prec_{1} y$ implies $x \prec_{2} y$.

A one-to-one map $\pi$ from $\mathbb{N}_{0}$ to $\mathbb{N}_{0}$ is said to be a permutation. For each $x$ in $X$, the composite map $x \circ \pi$ is a map from $\mathbb{N}_{0}$ to $Y$ and can be written as the infinite utility stream

$$
x \circ \pi=\left(x_{\pi(1)}, x_{\pi(2)}, \ldots, x_{\pi(n)}, \ldots\right) .
$$

The permutation $\pi$ is said to be finite if there exists a $t$ in $\mathbb{N}_{0}$, such that $\pi(n)=n$ for each $n \geq t$. The permutation $\pi$ is said to be fixed step if there exists a $t$ in $\mathbb{N}_{0}$, such that $\pi(\{1,2, \ldots, k t\})=\{1,2, \ldots, k t\}$ for each $k=1,2, \ldots$

Two of the most fundamental axioms in the area of ordering utility streams are the anonymity and the Pareto principle. The next definition lists some modifications.

Definition. Let $\precsim$ be a quasi-ordering in $X$.

- Strong Pareto: For each $x$ and $y$ in $X$, we have $x<y$ implies $x \prec y$.

- Intermediate Pareto: For each $x$ and $y$ in $X$, we have $x \leq y$ implies $x \precsim y$, and $x<y$ and $x_{i}<y_{i}$ for infinitely many $i$ in $\mathbb{N}_{0}$ implies $x \prec y$.

- Weak Pareto: For each $x$ and $y$ in $X$, we have $x \leq y$ implies $x \precsim y$, and $x \ll y$ implies $x \prec y$.

- Finite anonymity: For each finite permutation $\pi$ and for each $x$ in $X$, we have $x \sim x \circ \pi$.

- Fixed step anonymity: For each fixed step permutation $\pi$ and for each $x$ in $X$, we have $x \sim x \circ \pi$.

The strong Pareto axiom postulates sensitivity in each coordinate. The intermediate Pareto axiom postulates sensitivity in each infinite set of coordinates. When ranking subsets of $\mathbb{N}_{0}$, weak Pareto only requires that the full set $\mathbb{N}_{0}$ is strictly larger than the empty set. Fixed step anonymity is more demanding than finite anonymity, and imposes indifference between, for example, $(1,0,1,0, \ldots, 1,0, \ldots)$ and $(0,1,0,1, \ldots, 0,1, \ldots)$. Section 3 provides examples of fixed step anonymous and strong Paretian orderings in $X$. 


\subsection{The Axiom of Choice}

The Axiom of Choice $(\mathrm{AC})$ postulates for each nonempty family $\mathcal{D}$ of nonempty sets the existence of a function $f$ such that $f(S) \in S$ for each set $S$ in the family $\mathcal{D}$. The function $f$ is referred to as a choice function. AC does not provide an explicit way to construct such a choice function and provoked considerable criticism in the aftermath of Zermelo's formulation in 1904. The nonconstructive character of AC is further revealed by Dianonescu (1975) who showed that AC implies the law of the excluded middle. ${ }^{5}$

$\mathrm{AC}$ is $(i)$ consistent and (ii) independent: $(i) \mathrm{AC}$ can be added to the Zermelo-Fraenkel axioms of set theory without yielding a contradiction, ${ }^{6}$ and (ii) AC is not a theorem of the Zermelo-Frankel axioms (Fraenkel et al, 1973, Section II.4.2). Among the applications of AC, we mention Zorn's Lemma and the theorem of Hahn-Banach. AC also implies a number of paradoxes such as the decomposition of a sphere into a sphere of smaller size and the existence of a nonmeasurable set of real numbers.

Constructive mathematics rejects the law of the excluded middle and hence rejects AC. Beeson (1988 p42) lists a number of weaker choice principles that are generally accepted by constructivists. The Axiom of Dependent Choice (DC) is an example. Let $S$ be a nonempty set and let $R$ be a binary relation in $S$ such that for each $a$ in $S$ there is a $b$ in $S$ with $(a, b) \in R$. Then, DC postulates the existence of a sequence $\left(a_{1}, a_{2}, \ldots, a_{n}, \ldots\right)$ of elements in $S$ such that $\left(a_{k}, a_{k+1}\right) \in R$ for each $k=1,2, \ldots$.

For a thorough discussion of constructivism and its modifications, we refer to Beeson (1988); for a discussion of constructivism in economic theory, to Velupillai (2005); and for the history of the Axiom of Choice and its relation to the Zermelo-Fraenkel axioms of set theory, to Moore (1982) and Jech $(1973,1978)$.

\section{$3 \quad$ Ultrafilters and possibility results}

We recall the definition of an ultrafilter and show that its existence implies the existence of a finitely anonymous and strongly Paretian ordering in $X$.

Let $S$ be a set. A filter on $S$ is a nonempty family $\mathcal{F}$ of subsets of $S$ that satisfies

- the empty set is not in $\mathcal{F}$,

- if $A$ and $B$ are in $\mathcal{F}$, then $A \cap B$ is in $\mathcal{F}$,

- if $A$ is in $\mathcal{F}$ and $A \subseteq B$, then $B$ is in $\mathcal{F}$.

If the filter $\mathcal{F}$ on $S$ satisfies

\footnotetext{
5The law of the excluded middle states the truth of ' $P$ or not- $P$ ' for each proposition $P$ and can be used to claim the existence of certain objects without any hint to its construction. For example, the real number $c=\sqrt{2}^{\sqrt{2}}$ either is rational (in which case one sets $a=b=\sqrt{2}$ ) or is not rational (in which case one sets $a=c$ and $b=\sqrt{2}$ ). Conclude the existence of irrational numbers $a$ and $b$ for which $a^{b}$ is rational.

${ }^{6}$ Usually, ZF refers to the Zermelo-Fraenkel axioms. ZFC is obtained from ZF by adding AC to it.
} 
- for each $A \subseteq S$, either $A \in \mathcal{F}$ or its complement $S-A \in \mathcal{F}$,

then $\mathcal{F}$ is an ultrafilter. An ultrafilter is a filter that is maximal for inclusion. For example, the family of all cofinite subsets of $S$ (i.e. those subsets of $S$ whose complements are finite) is a filter on $S$. The family of all subsets of $S$ that contain a given element of $S$ is an ultrafilter on $S$ and is said to be principal. An ultrafilter is principle as soon it contains a finite set. An ultrafilter that is not principle is said to be free. The intersection $\cap_{\mathcal{F}} A$ of a free ultrafilter $\mathcal{F}$ is the empty set.

AC (rephrased as Zorn's lemma) implies the ultrafilter theorem: each filter over a set $S$ can be extended to an ultrafilter. The ultrafilter theorem is weaker than AC. Nevertheless, a free ultrafilter is a nonconstructive concept (Halpern, 1967; Jech, 1978).

For each $n$ in $\mathbb{N}_{0}$, let $\precsim_{n}$ be a Paretian and (finitely) anonymous ordering in $Y^{n}$. Let $\mathcal{F}$ be a filter on $\mathbb{N}_{0}$ that includes the family of all cofinite sets. Define the relation $\precsim_{\mathcal{F}}$ in $X$ as follows. For each $x$ and $y$ in $X$, we have

$$
x \precsim_{\mathcal{F}} y \quad \text { if and only if } \quad\left\{n \in \mathbb{N}_{0} \mid\left(x_{1}, x_{2}, \ldots, x_{n}\right) \precsim_{n}\left(y_{1}, y_{2}, \ldots, y_{n}\right)\right\} \in \mathcal{F} \text {. }
$$

The relation $\precsim_{\mathcal{F}}$ is reflexive (use $\mathbb{N}_{0} \in \mathcal{F}$ ); transitive (use the intersection property: if $A$ and $B$ are in $\mathcal{F}$, then $A \cap B \in \mathcal{F}$ ); strongly Paretian and finitely anonymous (use the fact that each cofinite set belongs to $\mathcal{F}$ ). In case the filter $\mathcal{F}$ contains the set $t \cdot \mathbb{N}_{0}=$ $\{t, 2 t, 3 t, \ldots, k t, \ldots\}$ for each $t$ in $\mathbb{N}_{0}$, then the relation $\precsim_{\mathcal{F}}$ satisfies fixed step anonymity. Furthermore, if $\mathcal{F}$ is an ultrafilter, then the relation $\precsim_{\mathcal{F}}$ is complete.

We apply this approach to the sequence of utilitarian orderings (see also Fleurbaey and Michel, 2003). Let $\mathcal{F}$ be a filter on $\mathbb{N}_{0}$ that includes the family of all cofinite sets. The quasi-ordering, defined by

$$
x \precsim U, \mathcal{F} y \quad \text { if and only if } \quad\left\{n \in \mathbb{N}_{0} \mid x_{1}+x_{2}+\cdots+x_{n} \leq y_{1}+y_{2}+\cdots+y_{n}\right\} \in \mathcal{F},
$$

extends the utilitarian criterion proposed by Basu and Mitra (2007). ${ }^{7}$ In case $\mathcal{F}$ is a free ultrafilter, the relation $\precsim_{U, \mathcal{F}}$ is a strongly Paretian and finitely anonymous ordering in $X$.

Next, let $\leq_{L, n}$ denote the leximin ordering in $Y^{n}$ and let $\mathcal{F}$ be a free ultrafilter on $\mathbb{N}_{0}$. The ultrafilter-leximin ordering in $X$, defined by

$$
x \precsim_{L, \mathcal{F}} y \quad \text { if and only if }\left\{n \in \mathbb{N}_{0} \mid\left(x_{1}, x_{2}, \ldots, x_{n}\right) \leq_{L, n}\left(y_{1}, y_{2}, \ldots, y_{n}\right)\right\} \in \mathcal{F},
$$

is strongly Paretian, finitely anonymous, and inherits the following properties of the leximin orderings:

- Hammond equity:

For each $x$ in $X$, for each pair $r$ and $s$ in $\mathbb{N}_{0}$ for which $x_{r}>x_{s}$, for each $y_{r}$ and $y_{s}$ in $Y$ such that $x_{r}>y_{r}>y_{s}>x_{s}$, the utility stream $y$ obtained from $x$ by replacing $x_{r}$ (resp. $x_{s}$ ) by $y_{r}$ (resp. $y_{s}$ ) is strictly preferred to $x$.

\footnotetext{
${ }^{7}$ Although the streams $x=(1,0, \ldots, 0, \ldots)$ and $y=\left(1 / 2,1 / 4, \ldots, 1 / 2^{k}, \ldots\right)$ distribute the same total utility, the utilitarian relation $\precsim U, \mathcal{F}$ ranks $x$ above $y$. This type of impatience can be avoided by developing a two-step procedure that first checks the convergency of $\sum_{i=1}^{\infty}\left(y_{i}-x_{i}\right)$ (see Lauwers and Vallentyne, 2004).
} 


\section{- Hammond equity for the future:}

For each $x_{1}, y_{1}, x$, and $y$ in $Y$ such that $x_{1}>y_{1}>y>x$, the infinite utility stream $\left(y_{1}, y, y, \ldots, y, \ldots\right)$ is strictly preferred to $\left(x_{1}, x, x, \ldots, x, \ldots\right)$.

The Hammond equity for the future axiom was introduced by Asheim and Tungodden (2005), further discussed in Asheim, Mitra, and Tungodden (2006), and states the following. If the present is better-off than the future and a sacrifice now leads to a uniform gain for all future generations, then such a sacrifice is desirable, as long as the present remains better-off than the future. Asheim, Mitra, and Tungodden explore different impossibility results arising from this axiom.

Finally, starting from the sequence of Lorenz quasi-orderings, this ultrafilter approach leads to a Lorenz quasi-ordering in the domain of infinite utility streams that satisfies the transfer principle (see also Bossert et al, 2006).

\section{Non-Ramsey sets}

We recall the Ramsey property, develop the main result, and position this result against those obtained by Zame (2007).

Let $I$ be an infinite set and let $n$ be a positive integer. Let $[I]^{n}$ collect all the subsets of $I$ with exactly $n$ elements. Ramsey (1928b) shows that for each subset $\mathcal{A}$ of $[I]^{n}$, there exists an infinite set $J \subset I$ such that either $[J]^{n} \subset \mathcal{A}$ or $[J]^{n} \cap \mathcal{A}=\varnothing$.

When $n$ is replaced by countable infinity, then Ramsey's theorem fails. There exists a subset $\mathcal{N}$ of $[I]^{\infty}$ such that for each infinite subset $J$ of $I$ the class $[J]^{\infty}$ intersects $\mathcal{N}$ and its complement $[I]^{\infty}-\mathcal{N}$ as well. Such a set $\mathcal{N}$ is said to be non-Ramsey. Building upon the work of Solovay (1970), Mathias (1977) showed that the existence of non-Ramsey sets does not follow from the axioms of Zermelo-Fraenkel (without AC). ${ }^{8}$

We develop an extra piece of notation. Let $i<j$ be two natural numbers in $\mathbb{N}_{0}$. The notation $\left[i, j\left[\right.\right.$ is a shorthand for the set $\{i, i+1, \ldots, j-1\}$. Let $S=\left\{n_{1}, n_{2}, \ldots, n_{k}, \ldots\right\}$ be an infinite subset of $\mathbb{N}_{0}$, we assume $n_{k}<n_{k+1}$ for each $k$. We connect two infinite sets to $S$ :

$$
\begin{aligned}
& S_{1}=\left[n_{1}, n_{2}\left[\cup \left[n_{3}, n_{4}\left[\cup \ldots \cup \left[n_{2 k-1}, n_{2 k}[\cup \ldots, \text { and }\right.\right.\right.\right.\right. \\
& S_{2}=\left[n_{2}, n_{3}\left[\cup \left[n_{4}, n_{5}\left[\cup \ldots \cup \left[n_{2 k}, n_{2 k+1}[\cup \ldots .\right.\right.\right.\right.\right.
\end{aligned}
$$

The triples $\left(S, S_{1}, S_{2}\right)$ play a crucial role in the main result. Recall that subsets of $\mathbb{N}_{0}$ are identified with infinite utility streams made up of zeros and ones.

Theorem. The existence of a complete, transitive, finitely anonymous, and intermediate Paretian relation in the set of (infinite) subsets of $\mathbb{N}_{0}$ entails the existence of a non-Ramsey set.

\footnotetext{
${ }^{8}$ More precisely, Solovay proposed a model in which ZF and DC are true and in which AC fails. Mathias showed that in this Solovay-model a non-Ramsey set does not exist. Hence, the existence of a non-Ramsey set is independent of ZF + DC.
} 
Proof. Let the ordering $\precsim$ in $\left[\mathbb{N}_{0}\right]^{\infty}$ satisfy intermediate Pareto and finite anonymity. Define $\mathcal{N}$ as follows:

$$
\mathcal{N}=\left\{S \mid S_{1} \prec S_{2}\right\},
$$

with $\left(S, S_{1}, S_{2}\right)$ as above. We show that for each infinite set $S=\left\{n_{1}, n_{2}, \ldots, n_{k}, \ldots\right\}$ in $\left[\mathbb{N}_{0}\right]^{\infty}$ there exists an infinite subset $T \subset S$ such either $S$ or $T$ belongs to $\mathcal{N}$ (the 'either-or' being exclusive). We distinguish two cases: $S \in \mathcal{N}$ and $S \notin \mathcal{N}$.

Case 1. The set $S$ is in $\mathcal{N}$, in other words $S_{1} \prec S_{2}$.

Consider the subset $T=\left\{n_{2}, n_{3}, \ldots, n_{k}, \ldots\right\}$ obtained from $S$ by cancelling out $n_{1}$. Then,

$$
\begin{aligned}
& T_{2}=\left[n_{3}, n_{4}\left[\cup \left[n_{5}, n_{6}\left[\cup \ldots \cup \left[n_{2 k-1}, n_{2 k}[\cup \ldots, \text { and }\right.\right.\right.\right.\right. \\
& T_{1}=\left[n_{2}, n_{3}\left[\cup \left[n_{4}, n_{5}\left[\cup \ldots \cup \left[n_{2 k}, n_{2 k+1}[\cup \ldots .\right.\right.\right.\right.\right.
\end{aligned}
$$

We have $T_{2} \cup\left[n_{1}, n_{2}\left[=S_{1}\right.\right.$ and $T_{1}=S_{2}$. Intermediate Pareto implies $T_{2} \precsim S_{1}$.

From $S_{1} \prec S_{2}$ and transitivity it follows that $T_{2} \prec T_{1}$.

Case 2.i. The set $S$ is not in $\mathcal{N}$, in particular $S_{2} \prec S_{1}$.

Again we consider the set $T=\left\{n_{2}, n_{3}, \ldots, n_{k}, \ldots\right\}$. In case $T_{1} \prec T_{2}$ we are done. Otherwise, we drop more elements from $S$. In particular, we drop $n_{4}$ and $n_{5}, n_{6}$ and $n_{7}$ until $n_{2 i}$ and $n_{2 i+1}$ to obtain the set $U=\left\{n_{2}, n_{3}, n_{2 i+2}, n_{2 i+3}, \ldots, n_{k}, \ldots\right\}$ such that:

$$
\begin{aligned}
& U_{2}=\left[n_{3}, n_{2 i+2}\left[\cup \left[n_{2 i+3}, n_{2 i+4}\left[\cup \ldots \cup \left[n_{2 k-1}, n_{2 k}[\cup \ldots, \text { and }\right.\right.\right.\right.\right. \\
& U_{1}=\left[n_{2}, n_{3}\left[\cup \left[n_{2 i+2}, n_{2 i+4}\left[\cup \ldots \cup \left[n_{2 k}, n_{2 k+1}[\cup \ldots .\right.\right.\right.\right.\right.
\end{aligned}
$$

The set $U_{2}$ includes $S_{1} \backslash\left[n_{1}, n_{2}\right.$ [ and the set $U_{1}$ is a subset of $S_{2}$.

The value of $i$ should be large enough to guarantee that the cardinality of $F=\left[n_{3}, n_{2 i+2}\right.$ [ exceeds the cardinality of $G=\left[n_{1}, n_{2}\left[\cup\left[n_{3}, n_{4}\left[\cup \ldots \cup\left[n_{2 i+1}, n_{2 i+2}[\right.\right.\right.\right.\right.$.

By finite anonymity $G$ can be embedded in $F$. The relation $U_{1} \prec U_{2}$ follows.

Case 2.ii. The set $S$ is not in $\mathcal{N}$, in particular $S_{1} \sim S_{2}$.

Drop the elements $n_{2}$ and $n_{3}, n_{6}$ and $n_{7}, n_{10}$ and $n_{11}$ and so forth $\left(n_{2+4 k}\right.$ and $n_{3+4 k}$ for each $k=0,1,2, \ldots)$. We obtain from $S$ the subset $T$ for which

$$
\begin{aligned}
& T_{1}=\left[n_{1}, n_{4}\left[\cup \left[n_{5}, n_{8}\left[\cup \left[n_{9}, n_{12}[\cup \ldots, \text { and }\right.\right.\right.\right.\right. \\
& T_{2}=\left[n_{4}, n_{5}\left[\cup \left[n_{8}, n_{9}\left[\cup \left[n_{12}, n_{13}[\cup \ldots .\right.\right.\right.\right.\right.
\end{aligned}
$$

The set $T_{1}$ includes $S_{1}$ and has infinitely many elements more than $S_{1}$. Also $S_{2}$ includes $T_{2}$ and has infinitely many elements more than $T_{2}$. Due to intermediate Pareto, we obtain $T_{2} \prec T_{1}$.

Starting from an element $S$ in $\mathcal{N}$ (resp. not in $\mathcal{N}$ ) we are able to find infinite subsets of $S$ not in $\mathcal{N}$ (resp. in $\mathcal{N}$ ). The set $\mathcal{N}$ is a non-Ramsey set.

This result confirms the conjecture of Fleurbaey and Michel. ${ }^{9}$ Consider two different utility levels, 0 and 1 . In the domain $\{0,1\}^{\mathbb{N}_{0}}$, intermediate Pareto notices an improvement

\footnotetext{
${ }^{9}$ The conjecture considers the continuous setting and weak Pareto. In contrast, we consider the discrete setting and impose intermediate Pareto.
} 
in case infinitely many generations increase their utility from 0 to 1 . Furthermore, finite anonymity is in the taxonomy of Fleurbaey and Michel (2003, Section 4.2) the weakest anonymity condition in an environment involving infinitely long utility streams. The result states that each quasi-ordering in the domain $\{0,1\}^{\mathbb{N}_{0}}$ that combines intermediate Pareto and finite anonymity is either incomplete or has no explicit description.

This result reveals a contradiction between the nonconstructive character of a nonRamsey set (a concept going back to Ramsey, 1928b) and Ramsey's (1928a) rejection to use a discount factor. In combination with Section 3, we conclude that the existence of a finitely anonymous and Paretian ordering is "in between" the existence of a free ultrafilter and the existence of a non-Ramsey set.

In order to position the result against those obtained by Zame, we distinguish the domains $[0,1]^{\mathbb{N}_{0}}$ and $\{0,1\}^{\mathbb{N}_{0}}$. Zame obtains the following results:

- Zame (2007, p197). The existence of a finitely anonymous and weakly Paretian ordering in $[0,1]^{\mathbb{N}_{0}}$ entails the existence of a nonmeasurable set.

- Zame (2007, p200). The existence of a finitely anonymous and strongly Paretian ordering in $\{0,1\}^{\mathbb{N}_{0}}$ entails the existence of a nonmeasurable set.

Since the restriction of a finitely anonymous and intermediate Paretian ordering in $[0,1]^{\mathbb{N}_{0}}$ to the restricted domain $\{0,1\}^{\mathbb{N}_{0}}$ is a finitely anonymous and intermediate Paretian ordering in $\{0,1\}^{\mathbb{N}_{0}}$, we obtain:

- The existence of a finitely anonymous and intermediate Paretian ordering in $[0,1]^{\mathbb{N}_{0}}$ entails the existence of a non-Ramsey set.

- The existence of a finitely anonymous and intermediate Paretian ordering in $\{0,1\}^{\mathbb{N}_{0}}$ entails the existence of a non-Ramsey set.

In the domain $[0,1]^{\mathbb{N}_{0}}$ Zame obtains the stronger result (weak Pareto is weaker than intermediate Pareto). In the domain $\{0,1\}^{\mathbb{N}_{0}}$ our result is stronger (intermediate Pareto is weaker than strong Pareto). Furthermore, within a discrete domain, it is impossible to strengthen our result through the weakening of the Pareto condition. To see this, consider the discrete set $Y_{\mathrm{d}}=\{0,1 \varepsilon, 2 \varepsilon, \ldots\}$ with $\varepsilon>0$. The map

$$
f: Y_{\mathrm{d}}^{\mathbb{N}_{0}} \longrightarrow \mathbb{R}: x=\left(x_{1}, x_{2}, \ldots, x_{n}, \ldots\right) \longmapsto \min \left\{x_{1}, x_{2}, \ldots, x_{n}, \ldots\right\}
$$

is well-defined and represents an anonymous and weak Paretian preference ordering on the discrete domain $Y_{\mathrm{d}}^{\mathbb{N}_{0}}$.

\section{References}

Asheim GB, Mitra T, Tungodden B (2006) Sustainable recursive social welfare functions. Memorandum 18/2006, Oslo University, Department of Economics 
Asheim GB, Tungodden B (2005) A new equity condition for infinite utility streams and the possibility of being Paretian. Memorandum 08/2005, Oslo University, Department of Economics

Basu K, Mitra T (2003) Aggregating infinite utility streams with intergenerational equity: the impossibility of being Paretian. Econometrica 71(5), 1557-1563

Basu K, Mitra T (2007) Utilitarianism for infinite utility streams: a new welfare criterion and its axiomatic characterization. Journal of Economic Theory 133, 350-373

Beeson MJ (1988) Foundations of constructive mathematics. Ergebnisse der Mathematik und ihrer Grenzgebiete 3.6. Berlin: Springer-Verlag

Bossert W, Sprumont Y, Suzumura K (2006) Ordering infinite utility streams. Journal of Economic Theory

Dianonescu R (1975) Axiom of choice and complementation, Proceedings of the American Mathematical Society 51(1), 176-178

Diamond PA (1965) The evaluation of infinite utility streams. Econometrica 33, 170-177

Fleurbaey M, Michel P (2003) Intertemporal equity and the extension of the Ramsey criterion. Journal of Mathematical Economics 39, 777-802

Fraenkel AA, Bar-Hillel Y, Levy A (1973) Foundations of set theory. Amsterdam: NorthHolland

Fudenberg D, Tirole J (1991) Game theory. Cambridge: MIT Press

Halpern JD (1967) The independence of the axiom of choice from the Boolean prime ideal theorem. Fundamenta Mathematicae 55, 57-66

Jech TJ (1973) The axiom of choice. Amsterdam: North-Holland

Jech TJ (1978) Set theory. New York: Academic Press

Lauwers L, Vallentyne P (2004) Infinite utilitarianism: more is always better. Economics and Philosophy 20, 307-330

Mathias ARD (1977) Happy families. Annals of Pure and Applied Logic 12, 59- 111

Moore GH (1982) Zermelo's axiom of choice: its origin, development, and influence. New York: Springer-Verlag

Ramsey FP (1928a) A mathematical theory of savings. Economic Journal 38, no. 192, 543-559

Ramsey FP (1928b) On a problem of formal logic. Proceedings of the London Mathematical Society, Series 2. 30.4, 338-384 
Rubinstein A (1979) Equilibrium in supergames with the overtaking criterion. Journal of Economic Theory 21(1), 1-9

Sidgwick H (1907) The methods of ethics. London: Macmillan

Solovay R (1970) A model of set theory where every set of reals is Lebesgue measurable. Annals of Mathematics 92, 1-56

Svensson LG (1980) Equity among generations. Econometrica 48, 1251-1256

Szpilrajn E (Marczewski E) (1930) Sur l'extension de l'ordre partiel. Fundamenta Mathematicae 16, 386-389

Velupillai KV (2005) The unreasonable ineffectiveness of mathematics in economics. Cambridge Journal of Economics 29(6), 849-872

Zame WR (2007) Can intergenerational equity be operationalized? Theoretical Economics $2,187-202$ 